\title{
THE RECOGNITION OF COMMON ORGANIC NERVOUS DISORDERS.
}

\author{
J. ST. C. ELKINGTON, M.B., M.R.C.P. \\ Assistant Physician, St. Thomas's Hospital.
}

THE diagnosis of disease depends upon the correct interpretation of facts derived from three sources-the history, the examination of the patient, and certain special investigations which may be necessary. Neurology affords no exception to this general rule ; indeed it exemplifies it very fully and calls upon these sources in the correct order of their importance : first history, then examination, and finally special investigation. Although no neurological diagnosis should be finally made until every piece of evidence to be obtained from the history and examination has been elicited and evaluated, it is of ten possible at the first moment of encounter with the patient to make a shrewd guess at the nature of his complaint and thereby to be enabled to direct fuller investigations along the most fertile channels. What is the material which is the subject of that instantaneous analysis which permits one to recognize many a clinical picture at sight with almost the certainty that one recognizes a cow or an oak tree? It is, surely, the patient's facial appearance, his speech, and the manner of his carriage and gait. It is true that these are all important "physical signs," but separately or together they often give the key to the diagnosis before anything more is known, and sometimes the vague impression they leave may lack confirmation at the end of examination and yet in the long run prove to have been correct. "Clinical instinct" in such a case is judged by those who believe in it to have triumphed over mere reason!

The best example of this is often provided by the Parkinsonian state, either senile or post-encephalitic, in its earliest stages. The patient perhaps complains that one hand feels weak, that one leg is stiff, or that it is difficult to walk up or down stairs; that he trembles, or is depressed, or dribbles, or has some other vague subjective trouble. Examination shows nothing to account for it. Close scrutiny shows a face of normal appearance with full voluntary movement; the limbs are strong enough and no tremor or disturbance of tone is detected; neither reflex nor sensory change can be found and the condition is considered to be "functional." But had the patient been studied as he approached from a distance, it might have been noticed that his face was a little fixed and lacking in expression, that the excitement of seeing the doctor caused fewer small facial movements than normal, that the eyes and head were lacking in mobility, that the trunk was slightly bent, and that the arms were held still instead of swinging naturally. The correct interpretation of these points would have saved the observer from missing the early manifestations of a grave and progressive disease.

The facial appearance is itself dependent upon a number of factors, the colour and texture of the skin, the hair, the facial and masticatory muscles, and the eyes and ocular muscles.

Subjective sensory phenomena, with or without symptoms of paraplegia, may often be recognized as due to subacute combined degeneration of the cord by the patient's facial appearance. The high colour of the cheeks may mask the underlying primary anæmia, but the red has a somewhat orange tint, and the rest of the face a slightly lemon or biscuit hue from the latent jaundice; examination shows the tongue to be 
perfectly smooth and lacking in papillæ, and often it is fissured and may be sore. The hair is frequently prematurely white.

It is well recognized that acromegaly, even in its earliest stages, produces characteristic changes in the face. There is a coarsening of the texture of the skin and hair, increase in the frontal and malar eminences and growth of the lower jaw with increasing obtuseness of its angle and spacing of the contained teeth. The nose and tongue share this increase in size, the voice grows deeper and gruffer. An acromegalic man spontaneously made this statement : "When I was young I had a very good tenor voice, but it gradually became baritone and now I sing bass."

It is less well known that chromophobe adenomata of the pituitary produce a change in the face almost as characteristic and easily recognizable. The skin grows pale till it may be of uniformly waxen hue. It becomes dry and its texture softens until it resembles that of a child. The eyebrows, and in men the beard, become dry and scanty in amount. The subcutaneous tissues are slightly puffy, giving a myxœdematous appearance which is enhanced by the sleepy mental state of the sufferer. The hands are soft and puffy with slender tapering fingers.

Ptosis is a sign of nervous disease which may quickly attract attention. Deeply engraved furrows on the forehead, from constant wrinkling of the brow in an attempt to compensate for the drooping of the lids, show it to be of long duration. Combined with irregular and unequal pupils reacting imperfectly, such long-standing ptosis is usually significant of neurosyphilis.

A very characteristic expression is acquired by persons suffering from myasthenia gravis. Ptosis is usual, but attempts to compensate for it by overaction of the frontales, such as are seen in tabes, are often unavailing owing to the weakness of the facial muscles; the patient throws his head back to get a better view from beneath his drooping eyelids, but soon wearies of this attitude and is forced to support his head with his hand. At times, especially when eating, the masseters become too weak to support the jaw against gravity and it is held up with the hand. The facial expression is mournful, the smile peculiarly "snarling" in quality. Frank but very variable oculomotor paralyses are often present, and the voice is husky or nasal in quality and grows more so as speech continues and fatigue develops.

Myopathic patients also wear a sad expression, but ptosis and oculomotor palsies are never seen. The facial muscles are weak, especially the orbiculares of the eyes and mouth. The lips protrude, the smile is wide and transverse and the patient is unable to whistle or to smoke a cigarette. Speech may be indistinct from defective production of the labial sounds. The eyes cannot be tightly shut and often remain open during sleep.

Unilateral facial weakness is not infrequently one of the earliest physical signs of nervous disease. When of the supranuclear type it is first seen as a slight defect in movement of the corner of the mouth in showing the teeth or smiling, when of infranuclear type it more often shows itself first in the upper face as a widening of the palpebral fissure or defective blinking. The latter type of weakness may be combined with tonic spasm which at first conceals it, or with clonic spasm; both these phenomena usually signify an irritation of the facial nerve trunk. 
The highly complex function of speech may be deranged in a number of ways. Its subject matter may be inappropriate, indicating an abnormality in the mind which directs it. The construction of the sentences and the manipulation of speech symbols may be faulty, giving rise to aphasia, or the defect may be in the executive function of the larynx and pharynx leading to dysarthria.

Perhaps the most constant mental change encountered in organic nervous disease is that found in disseminate sclerosis. A predominant mood of abnormal cheerfulness coexists with a marked emotional instability. Temperamentally these patients resemble a pleasant April day, smiles and tears succeed each other with bewildering rapidity, but on the whole the smiles have the better of it. The picture of an almost paraplegic patient dragging his legs along with painful slowness, supported by two sticks but wearing a happy smile and asserting that he never felt better in his life, is almost pathognomonic of this disease.

The constant mental defect found in organic disease of the brain, be this neoplastic, vascular or inflammatory, is some degree of dementia with consequent decrease in attention, comprehension and self-control. The widely differing pictures which this change produces owe their variety to the diversity of the underlying temperaments upon which the dementia is operating rather than to the particular pathological process at work. The melancholic individual becomes depressed, the self-assertive one truculent, and so on.

The degree of dementia appears to depend upon the quantity of brain substance destroyed rather than upon its site, and upon damage of the subcortical white matter rather than of the grey.

Aphasia in its most typical form can hardly be mistaken for any other condition, but some varieties are less readily recognized.

The incoherent stream of meaningless syllables poured out with increasing speed and excitement by the "jargon" aphasic is surprisingly often mistaken for a symptom of purely psychic origin by those unfamiliar with the condition.

In some cases of aphasia the defect may be so entirely confined to the execution of speech, without any disturbance of the mental manipulation of speech symbols, that the patient is thought to have a stammer or some kind of dysarthria.

In others the receptive side of the speech mechanism is chiefly disorganized, leading to so much confusion and mental impairment that the aphasic nature of the condition may be quite overlooked.

The former condition is usually associated with lesions far forward in the left hemisphere, the latter with those far back.

Correct articulation depends upon the integrity of the upper motor neurone pathway from the cortex to the bulbar nuclei, upon the lower motor neurone pathway comprising the bulbar nuclei, the nerves proceeding from them and the muscles innervated and upon correct co-ordination through the influence of afferent impulses from the muscles themselves and through the cerebellar system.

The slow, stiff, "spastic" speech is the rule in cases of double hemiplegia from multiple vascular lesions, and in cases of amyotrophic lateral sclerosis in which the maximal incidence of the disease is upon the upper motor neurones. 
The weak, slurring, indistinct articulation characteristic of a lower motor neurone dysarthria is best illustrated by cases of progressive bulbar palsy.

In lesions of the peripheral nerves of the speech mechanism and of the muscles themselves, the functions of the palate and the abductors of the vocal cords usually suffer first, and we find the nasal voice so common in diphtheritic paralysis and myasthenia gravis, or the husky voice of patients with an abductor palsy.

Cerebellar dysfunction affects speech as it affects other motor functions, by producing inco-ordination. The speech instead of flowing in a smooth and modulated stream becomes irregular in force, tone and rhythm. It becomes explosive, words falling over each other at one moment and being unduly spaced at the next. Such dysarthria is heard in disseminate sclerosis, in Friedreich's ataxy, in lesions involving the cerebellar connections in the medulla, or of the efferent cerebellar pathways to the mid-brain.

Gait, like speech, is no simple process. Not only have the legs to support the bodyweight and carry it forward, but many muscles have to co-operate in the production of the associated movements necessary to preserve balance. The same essential elements are involved, the functions of the upper motor neurone path, of the lower motor neurone path including the peripheral nerves and muscles, and the sensory and cerebellar elements of co-ordination.

Unilateral disease of the pyramidal pathway above its decussation leads to a spastic weakness of the body on the opposite side. The affected leg tends always to take up a posture of full extension, and in order to prevent the foot from dragging along the ground the leg is circumducted at the hip in walking, giving rise to the characteristic hemiplegic gait.

Disease of both pyramidal tracts in the spinal cord produces a similar condition, but in both legs, and the gait of the spastic paraplegic most frequently seen in disseminate sclerosis results.

Disease of the peripheral nerves usually first causes weakness in the dorsiflexors of the ankles, giving rise to "foot-drop." In walking, in order that the dropped foot shall clear the ground the patient lifts his whole leg higher than normal in a so-called "steppage" gait. As sensation from the lower extremities is usually defective in these cases the feet are likely to be replaced on the ground in a clumsy, inco-ordinate manner.

Primary disease of the muscles occasionally first involves the anterior tibial and peroneal groups of muscles producing a very similar picture of "foot-drop." More often, however, in myopathies, the first muscles to be affected are those of the trunk and proximal portions of the limbs. The pelvis is then insecurely held in relation to the spine and femora and the gait becomes "waddling" in character. Great difficulty is experienced in rising from the lying or sitting posture.

Pure sensory inco-ordination is seen at its fullest in advanced cases of tabes, but a very similar picture may be seen in subacute combined degeneration of the cord, and in local spinal lesions in which the posterior columns are chiefly involved. The legs may be quite strong, but the afferent stimuli from the muscles and joints carrying information about the position of the limbs in space no. longer reach the reflex centres, and gait instead of being an orderly proceeding becomes a game of blind man's buff. 
The feet are lifted too high or not high enough, one leg is thrown out from its line of march or across the path of its fellow, the feet are replaced on the ground in the wrong place and often with a thud. Walking may become quite impossible without any weakness being present.

Inco-ordination of a different kind is seen in cerebellar lesions. The afferent pathways are intact but the orderly execution of simultaneous and successive movements is impaired. The legs are held wide apart to give a broader base, one or both legs tends to deviate to right or left of its line of march. Any slight tendency to lose balance is corrected late and with difficulty and often too violently, and the gait becomes staggering and "drunken" in quality.

Such inco-ordination of gait is met with in chronic disease of the cerebellum such as tumours or degenerations, in degenerations of the cerebellar tracts as in Friedreich's disease and the other hereditary ataxies. In acute cerebellar lesions the ataxy is usually too intense to permit even an attempt at walking.

In many nervous diseases the associated movements seen in normal progression are diminished. In hemiplegias and in Parkinsonian states the arm instead of swinging naturally is held stiff and still. In cerebellar disorders and in chorea the affected arm is seen to hang passively and to swing like a pendulum under the influence of gravity rather than by its own associated movement.

In other cases involuntary movements are seen. The rhythmic tremor of paralysis agitans, the irregular jerky movements of chorea, the head nodding of Friederich's disease and the squirming movements of athetosis seen in juvenile hemiplegia may all be accentuated during the act of walking.

The foregoing are some of the pieces of information that can be elicited from a patient with nervous disease at first sight and before any history has been taken or any detailed examination made. They may be of inestimable value, but the method of quick diagnosis should be used with great caution, and first impressions should be discarded without hesitation if they will not stand the test ot careful examination and reasoned criticism.

\section{DEFORMITIES OF THE FOOT IN CHILDREN.}

\section{The Treatment of those Types due to Affections of the Nervous System.}

\section{BY B. WHITCHURCH HOWELL, F.R.c.s.}

THE following types will be discussed in this article :-

(I) Anterior poliomyelitis.

(2) Spastic paralysis.

(3) Fredreich's ataxia.

(4) Pseudo-hypertrophic muscular paralysis.

(1) Anterior Poliomyelitis.

The clinical picture of the acute stage is too well known to be enlarged upon. The cardinal principles of treatment are to rest the weak or paralysed muscles in the position of optimum utility, preferably in simple splints in the prone position, or in plaster of 